\title{
Cirrosis hepática: perfil epidemiológico y calidad de vida. Hospital Teodoro Maldonado Carbo. Período 2014 $-2015$
}

\section{Liver cirrhosis: epidemiological profile and quality of life. Hospital Teodoro Maldonado Carbo Hospital. Period 2014 - 2015.}

Katherine Andrea Flores Poveda. ${ }^{1}$, Jorge Emilio Morante Arias. ${ }^{2}$, Denisse Lissette Flores Subia. ${ }^{3} \&$ Alexander Manuel Méndez Castro ${ }^{4}$.

\section{Abstract. \\ DOI: https://doi.org/10.33262/cienciadigital.v3i4.936}

Liver cirrhosis is a chronic, terminal disease of the liver, that affects its structure and physiology. The frequent causes are the alcohol consumption (90\%); viral hepatitis; hepatic steatosis; metabolic diseases, etc. In Ecuador, despite the existence of several degree studies to support this data, this study aims to analyze the current situation in our hospital environment in order to create a pillar capable of sustaining an epidemiological profile of risk when defining which patients have a tendency to complicate their picture and to overshadow their quality of life due to the complications they may present. It is nonexperimental, descriptive, retrospective, cross-sectional a study where $151 \mathrm{HC}$ of patients entered with diagnosis of $\mathrm{CH}$ in HTMC were reviewed, using like variables: patient data, habits, complications, to describe to the profile epidemiologist; and MELD and Child-Pugh scales to describe its quality of life. Our collected data of $\mathrm{HC}$ registered show a tendency towards advanced stages, with greater hepático commitment with a survival in 2 years of as soon as $35 \%$ according to the Child-Pugh scale and by MELD Scale are oriented towards results of 10-19 that correspond to a survivence in 3 months over 52,6\%; the antecedents of alcoholism and bad nutritional habits constitute sociodemographic profile. The lack of information in

\footnotetext{
${ }^{1}$ Hospital General Monte Sinaí, Guayaquil, Ecuador, Email: katitaflores_p@ hotmail.com

${ }^{2}$ Hospital General Monte Sinaí, Guayaquil, Ecuador, Email: Emiliox2102@ hotmail.com

${ }^{3}$ Hospital General Monte Sinaí, Guayaquil, Ecuador, Email: denisse64@hotmail.com

${ }^{4}$ Medical Master S.A, Guayaquil, Ecuador. dante_2011ammc@hotmail.com
} 
the HC made difficult its exhaustive analysis, nevertheless we can define to mortality like pounding fundamental in the quality of life, we recommended future investigations to extend the margin search to strengthen to greater number of patients within the population.

Keywords: Cirrhosis, Risk Factors, Alcohol, Eating Habits.

\section{Resumen.}

La cirrosis es una enfermedad crónica, terminal del hígado, que afecta su estructura y fisiología. Las causas frecuentes son el consumo de alcohol (90\%); las hepatitis virales; hígado graso; enfermedades metabólicas, etc. En Ecuador a pesar de existir varios trabajos de titulación que respalden este dato, este estudio pretende describir la situación actual en nuestro medio hospitalario para crear un pilar capaz de sostener un perfil epidemiológico de riesgo al definir que pacientes tienen tendencia a empeorar su cuadro y ensombrecer su calidad de vida por las complicaciones que pudieran presentar. Es un estudio no experimental, descriptivo, retrospectivo, transversal dónde se revisaron $151 \mathrm{HC}$ de pacientes ingresados con diagnóstico de $\mathrm{CH}$ en el HTMC, utilizando como variables: datos de filiación, hábitos, complicaciones, para describir el perfil epidemiológico; y escala MELD y Child-Pugh para describir su calidad de vida. Nuestros datos recolectados de $\mathrm{HC}$ registradas muestran una tendencia de $\mathrm{CH}$ hacia estadios avanzados, con compromiso hepático mayor con una supervivencia en 2 años de apenas el 35\% según la escala de Child-Pugh y por MELD se orienta hacia puntaciones de 10-19 que corresponde a una sobrevida a los 3 meses del 52.6\%; los antecedentes de alcoholismo y malos hábitos alimenticios constituyen el perfil sociodemográfico predominante. La falta de información en las HC dificultó su análisis exhaustivo, sin embargo, podemos definir a la mortalidad como pilar fundamental en la calidad de vida, recomendamos para futuras investigaciones ampliar el margen de búsqueda para afianzar a mayor número de pacientes dentro de la población.

Palabras claves: Cirrosis, factores de riesgo, alcohol, hábitos alimenticios.

\section{Introducción.}

La cirrosis hepática $(\mathrm{CH})$ es una entidad clínica que abarca todos los estratos sociales, llegando al punto de considerarlo un problema de salud pública, ya que la tendencia ha aumentado levemente a escala global. Diversos factores se pueden disociar para entender el origen de la prevalencia de la cirrosis, encasillando en un perfil epidemiológico definido; no obstante, en la mayoría de los casos, los datos son sesgados y no dan una certera información que relacione a los pacientes con su posible causa. Además, la cirrosis es la etapa final de las patologías del hígado que conducen a estadios avanzados de disfunción, produciendo severas complicaciones que ensombrecen su calidad de vida, que lamentablemente lleva a cabo 
producto de diversas determinantes socioculturales, o por el descuido o falta de concientización del paciente con respecto a su enfermedad.

Según estadísticas mundiales la causa más frecuente como etiología de cirrosis hepática, es la ingesta excesiva de bebidas alcohólicas con un $90 \%$ de los casos, seguido por esteatosis hepática y por hepatitis vírica. (web, 2016). No obstante, en la actualidad y a escala global, la mortalidad por cirrosis hepática ha disminuido, debido a cambios en los hábitos o por un manejo adecuado del tratamiento en dichos pacientes. En los países europeos, la cirrosis hepática tuvo un aumento de la mortalidad desde 1959 hasta los años 90, que luego fue disminuyendo posteriormente en la última década. En los Estados Unidos, la tendencia encontrada de consumo de alcohol estimado por estadísticas referentes a la producción de bebidas alcohólicas, demostró que sólo el 40-60\% de la población son consumidores.

En Ecuador, la Cirrosis Hepática ocupa el 7mo lugar en causas de mortalidad, según datos del INEC en el año 2014; donde las enfermedades coronaries isquémicas ocupan el primer lugar, seguido por la diabetes mellitus, enfermedad cerebrovascular, enfermedad hipertensiva, infecciones respiratorias bajas, accidentes de transporte terrestre; en orden descendente. En ese año se reportaron 2005 fallecidos por enfermedad hepática dejando una tasa de mortalidad 12,71, que al comparar según el género de los afectados su varianza es marcada 14,85 para hombres y 10,76 para mujeres. Esta patología se encuentra más ligada a la edad avanzada, con prevalencia elevada pasadas los 50 años. (Moncayo, 2014) En nuestro país, a pesar de existir varios trabajos relacionados, no existen estadísticas publicadas ni verificadas en relación a revistes indexadas, por ello este estudio pretende analizar la situación actual en nuestro medio hospitalario con el fin de crear un pilar capaz de sostener un perfil epidemiológico de riesgo al momento de definir que pacientes tienen tendencia a complicar su cuadro y ensombrecer su calidad de vida por las complicaciones que pudieran presentar.

El propósito del presente estudio es determinar el perfil epidemiológico de los pacientes diagnosticados con Cirrosis Hepática $(\mathrm{CH})$ en nuestro entorno hospitalario y poder determinar la calidad de vida que ellos presentan, mediante el estudio de las características sociodemográficas de cada uno y su nivel en score MELD y Child-Pugh, dando como resultado un estudio de corte transversal cualitativo retrospectivo no experimental.

\section{Metodología}

\section{Etiología}

Dentro de las causas reconocidas de esta enfermedad, se menciona a la ingesta de bebidas alcohólicas como la principal causa, seguida de infecciones por virus de Hepatitis C y Hepatitis B, hígado graso no alcohólico, cirrosis biliar primaria o secundaria, hemocromatosis y enfermedad de Wilson. Cabe mencionar que un paciente con cirrosis debe 
realizarse estudios imagen lógicos para descartar un hepatocarcinoma pues pueden desarrollar en un 30\% de los casos esta enfermedad subyacente. (Phillip S. Ge, 2016)

\section{Epidemiología y antecedentes}

En el 2003 en la Revista Americana de la Sociedad de Hepatología, recalcó que el 44\% de las muertes por enfermedades hepáticas se vinculaban con el hábito de ingesta de bebidas alcohólicas, cifra que en el 2015 llego a los 3,3 millones. Con una prevalencia de 250 casos por cada 100.000 habitantes en países de primer mundo. (Usiña \& Carrera, 2013)

La cirrosis hepática es una causa importante de morbilidad y mortalidad en el mundo. Se estiman 800.000 muertes anuales a nivel mundial. La cirrosis hepática en Europa y EE. UU. Posee una prevalencia de alrededor de 250 casos anuales por cada 100.000 personas. En los hombres la prevalencia es dos veces mayor que en las mujeres. Según diferentes estimaciones de los expertos, la prevalencia de la cirrosis en España es de entre 1 y 2\% de la población, siendo más frecuente en varones a partir de los 50 años.

En algunos países de América Latina, como Chile y México, la cirrosis hepática ocupa, entre el $5^{\circ}$ y $6^{\circ}$ lugar como causa de muerte general. En México la cirrosis hepática es la tercera causa más común de mortalidad en hombres y la séptima en mujeres. Se ha descrito que la mortalidad por cirrosis hepática varía entre 11.6 a 47.4 por 100,000 habitantes, encontrándose la mayor mortalidad en el área central del país. El promedio de edad es de $50.3+12.0$ años. Chile reporta la cirrosis hepática como la $5^{\mathrm{a}}$ causa de muerte a pesar de que las tendencias de hospitalización y de muerte se modificaron en la última década del siglo XX.

En el 2013 Ecuador público a través del INEC, que la cirrosis hepática ocupaba la 7ma causa de mortalidad en nuestro país con cerca del 12,71 como razón de mortalidad. Es decir, después de la HTA y DM como enfermedades crónicas y degenerativas, la cirrosis sigue en prevalencia y victimas anuales. Donde los pacientes en un entorno social donde el alcohol es del folclor, se convierten la ingesta de este producto en determinante en el momento del desarrollo de esta patología. (web, 2016)

Sin embargo, a pesar de los mencionados datos mundiales capaces de definir causa específica, en nuestro entorno no existen estadísticas hospitalarias publicadas en revistes indexadas y la falta de información sobre la calidad de vida se convierten en el objetivo central de este proyecto al querer definir los factores de riesgo asociados en la población diagnosticada con cirrosis hepática y la calidad de vida q ellos presentan sea por la probabilidad de mortalidad o reingresos por complicaciones asociadas. (Octavio Campollo, 1997,).

\section{Anatomía patológica y fisiopatología}

La cirrosis es un proceso difuso que compromete el parénquima hepático de forma fibrótica, con regiones de regeneración, que le dan una apariencia heterogénea. Es decir, existirán 
zonas necróticas difusas asociadas a nódulos de regeneración; lo que fomenta la fibrosis y creación de septos interlobulillares que logran disminuir la perfusión sanguínea e intercambio de sustratos por lo que se marca en este punto, una marca de no retorno hacia la cirrosis hepática.

Este fenómeno produce un aumento de la relación de la presión portal y la vena cava mayor a $5 \mathrm{mmHg}$, que se conoce como hipertensión portal y las complicaciones que pueden desencadenar como ascitis, síndrome hepatorrenal, peritonitis bacteriana espontanea, encefalopatía hepática, hemorragia por varices esofágicas entre otros. (Bueya \& Mateosb, 2012) (D. Rincóna, 2016)

\section{Clasificación clínica}

La clasificación de la cirrosis hepática puede darse mediante criterios clínicos según la conferencia de Baveno IV; siendo estable y no estable. Al hablar de factores pronósticos en este punto, la fase conocida como cirrosis compensada está determinada por el grado de hipertensión porta, plaquetopenia, presencia o ausencia de varices esofágicas; mientras que en la cirrosis descompensada la ascitis, hemorragia varicial, encefalina hepática y presencia de ictericia; que marcan la supervivencia para el primer año del $61 \%$ y en el segundo año el $50 \%$.

Dividiéndose la primera en estadio I sin varices sin ascitis, y estadio II varices sin ascitis; mientras que la segunda como no estable, estadio III ascitis con varices esofágicas sin ser hemorrágicas, IV hemorragia por varices con ascitis. (D. Rincóna, 2016)

\section{Pronóstico}

Mientras la causa lesiva a nivel del parénquima se mantenga, la lesión continuara progresando. Por lo que dentro del manejo inicial consiste en contrarrestar la lesión causante de la cirrosis, que en muchos casos permite un correcto control de la enfermedad y manejo de manteamiento. Caso contrario el estadio final amerita un trasplante. (L. Téllez*, 2016)

\section{Calidad de vida en el paciente cirrótico}

Dado que la calidad de vida está ligada a las probabilidades de vida y conciencia que el paciente tome de su enfermedad, en el 2016 se publicó el nivel de sobrevida según el grado de compromiso de los pacientes, es decir según el estadio clínico en el que se encontraba. Los pacientes en estadio I con una cirrosis compensada anualmente pudieran presentar 4,4\% de probabilidades de complicarse, pero si esto no sucede su probabilidad de mortalidad en el primer año es del 1\%; aquellos con grado II les siguen con un 3,4\%; en el caso de cirrosis descompensada en estadios III su probabilidad se eleva al 20\%; mientras que en grado IV esta se eleva a más del $57 \%$. Por lo cual si decidimos ver la calidad de vida de estos pacientes 
bastaría con determinar el nivel de progreso de los pacientes o estadio de los mismos y según se determinaría. (Bueya \& Mateosb, 2012)

\section{Medición de la mortalidad de cirrosis hepática: escala CHILD-PUGH y escala MELD.}

La clasificación modificada Child-Pugh de severidad de enfermedad hepática se realiza de acuerdo al grado de ascitis, las concentraciones plasmáticas de bilirrubina y albúmina, el tiempo de protrombina, y el grado de encefalopatía. Una puntuación total de 5-6 es considerado grado A (enfermedad bien compensada); 7- 9 es grado B (compromiso funcional significativo); y $10-15$ es grado $\mathrm{C}$ (enfermedad descompensada). Estos grados se correlacionan con una sobrevida del paciente al año y a los 2 años.

Para medir el pronóstico de los pacientes se emplea diferentes mecanismos de evacuo, invasivos y no invasivos. Dentro de los invasivos tenemos la biopsia como elemento determinante por su tasa de especificidad; para los no invasivos mediante fórmulas y base de laboratorio se pudieran emplear fórmulas como: FORNS, MADDREY; MELD SCORE; GLASGOW ALCOHOLIC HEPATITIS SCORE; APRI, que permiten medir el nivel de compromiso de la función hepática y el posible pronóstico de cada paciente.

\begin{tabular}{|c|c|c|c|}
\hline \multicolumn{4}{|c|}{ Clasificación de Child-Pugh } \\
\hline Puntuación & 1 & 2 & 3 \\
\hline Ascitis & Ausente & $\begin{array}{l}\text { Controlada con } \\
\text { diuréticos }\end{array}$ & $\begin{array}{l}\text { No controlada con } \\
\text { diuréticos }\end{array}$ \\
\hline Encefalopatía & Ausente & Grado $102 / 4$ & Grado $3 \circ 4 / 4$ \\
\hline Bilirrubina & $<2$ & $2-3$ & $>3$ \\
\hline Albúmina & $>3,5$ & $2,8-3,5$ & $<2,8$ \\
\hline INR & $<1,7$ & $1,8-2,3$ & $>2,3$ \\
\hline \multicolumn{4}{|c|}{$\begin{array}{l}\text { Puntuación total: suma de los } 5 \text { items. } \\
\text { Grado A (cirrosis compensada): } 5 \text { o } 6 \text { puntos. } \\
\text { Grado B (cirrosis moderadamente descompensada): } 7 \text { a } 9 \text { puntos. } \\
\text { Grado C (cirrosis gravemente descompensada): } 10 \text { a } 15 \text { puntos. }\end{array}$} \\
\hline
\end{tabular}

Figura 1. Clasificación de Child-Pugh

Fuente: MEDICINE, 2016. 


\begin{tabular}{l|c|c|c|}
\hline Variable & 1 punto & 2 puntos & 3 puntos \\
\hline Edad (años) & $<50$ & $\geq 50$ & - \\
Leucocitos (109/L) & $<15$ & $\geq 15$ & - \\
Urea (mmol/L) & $<5$ & $\geq 5$ & - \\
INR & $<1.5$ & $1.5-2.0$ & $>2.0$ \\
Bilirrubina (mcmol/L) & $<125$ & $125-250$ & $>250$ \\
Para convertir Urea de mg/dL a unidades del Sistema Internacional (mmol/L), se debe multiplicar \\
$\begin{array}{l}\text { el valor de urea en } \mathrm{mg} / \mathrm{dL} \text { por la constante } 0.1665 \text {. Para convertir bilirrubina de } \mathrm{mg} / \mathrm{dL} \text { a unidades } \\
\text { del Sistema Internacional (mcmol/L), se debe multiplicar el valor de bilirrubina en mg/dL por la } \\
\text { constante 17.104. INR= International normalized ratio. }\end{array}$
\end{tabular}

Figura 2. Índice de Glasgow para Hepatitis alcohólica. Fuente: Revista Chilena de Hepatología, 2015.

Mediante la aplicación de estos scores nos permitirán establecer la calidad de vida y pronóstico de cada paciente, de esta manera al recolectar los datos, se obtendrían una base específica (Robert S. O’Shea, 2010)

A pesar de que estos scores son empleados ampliamente para determinar la calidad de vida de los pacientes y pronóstico de cada uno, la gran mayoría son empleadas con pacientes con hepatitis.

El score MELD emplea los parámetros como Bilirrubina, INR, Creatinina, Sodio y si ha recibido diálisis o no. Los resultados pueden predecir o aproximar el pronóstico de vida en los siguientes 3 meses por lo cual con un puntaje de 9 se llega 1.9\%, 10 a 19 con 6\%, 20 a 29 con $19,6 \%$, 30 a 39 con 52,6\% y 40 la mortalidad se eleva a 71,3\% en los próximos tres meses. Este score se convierte en la base para determinar el pronóstico en nuestros pacientes ingresados con diagnóstico de cirrosis hepática.

\section{Manejo inicial y tratamiento en pacientes cirróticos.}

En el manejo de la cirrosis, se basa en pilares nutricionales y farmacológicos. En el aspecto de nutrición la dieta debe ser hiperproteica (sin exceder 1,5gr/Kg día), hipo sódico, libre de grasa. El empleo de antihipertensivos de tipo betabloqueante se convierte en la siguiente etapa con el objeto de evitar una complicación mayor hacia la hipertensión portal. (Shah, 2012) 


\section{Complicaciones en pacientes cirróticos}

Cuando el gradiente de presión portal se eleva, las complicaciones en virtud de la cirrosis hepática se presentan; ascitis, encefalopatía hepática, sangrado varicial, síndrome asociados a nefropatías. La descompensación hemodinámica se inicia con la acumulación de elementos nitrogenados en sangre, hipotensión sistémica e hipertensión portal, que mediante la contra regulación de mecanismos hipertensivos (entre ellos el sistema renina angiotensina aldosterona), aumentan el nivel de sodio y retención de agua. Esto último conlleva a la ascitis y descompensación cardiaca por la baja precarga resultante. A su vez si este factor no es controlado llega al conocido síndrome hepatorrenal asociándose o no a ascitis bacteriana espontanea por la fuga de bacterias desde el intestino hacia la cavidad abdominal que ahora acumula líquido.

Todo esto es capaz de terminar en una descompensación hemodinámica que pudiera provocar falla multiorgánica y llegar a la muerte del paciente. (Phillip S. Ge, Treatment of Patients with Cirrhosis, 2016) Cualquier complicación que pudiera desarrollar un paciente con hipertensión portal, ensombrece el pronóstico de los pacientes, tomando como ejemplo a la ascitis su mortalidad se eleva en un 11,6\%; con la presencia de hemorragia varicosa $3,78 \%$, hepatocarcinoma con un $6,1 \%$.

Una de las complicaciones que pueden ser mortales son peritonitis bacteriana espontánea (PBE) que se presenta en un 10-30 \% de los enfermos con ascitis. Está causada por la infección del líquido ascítico sin que exista una fuente de infección visible en la cavidad abdominal. Probablemente se produce a consecuencia de la translocación de bacterias desde la luz del tracto digestivo y de la alteración de la actividad antibacteriana del líquido ascítico. Las bacterias aisladas con mayor frecuencia son (70 \%): Escherichia coli, Enterococcus faecalis, Enterobacter, Serratia, Klebsiella, Proteus, Pseudomonas.

El sangrado del tracto digestivo es la complicación más probable en enfermos con cirrosis hepática y el más significativo clínicamente, manifestándose en forma de sangrado por varices esofágicas ( $10 \%$ de todas las causas de sangrado del tracto digestivo superior), que es consecuencia de la circulación colateral en la hipertensión portal. El riesgo de hemorragia de las varices esofágicas es de $\sim 30 \%$ a los 2 años desde su diagnóstico por endoscopia. En $\sim 10 \%$ el lugar de sangrado no son las varices esofágicas, sino las venas gástricas (sangrado por varices subcardiales, difíciles de diagnosticar y de tratar). Mucho menos frecuentemente es el sangrado de las varices del tracto digestivo inferior (p. ej. hemorroides) y sus consecuencias no son tan graves. Su tratamiento es inmediato e intrahospitalario.

La encefalopatía hepática es el conjunto de trastornos funcionales del SNC en el curso de las enfermedades hepáticas severas agudas o crónicas, probablemente como resultado de la 
acción de neurotóxicas endógenas (amoníaco, mercaptanos, ácidos grasos de cadena corta y media, fenoles), de la presencia de falsos neurotransmisores o de una activación excesiva del sistema gabanérgico. Se presenta como trastornos del comportamiento, del estado de ánimo, de la personalidad, de las funciones intelectuales, de la conciencia y de la actividad neuromuscular, de un grado de intensidad variable.

Todas las patologías antes mencionadas que acompañan a la cirrosis hepática son predictores de su calidad de vida. Por tal motivo se debe evitar que un paciente de este tipo llegue a descompensarse y desarrollar cualquier tipo de complicación que puede costarle la vida. Es decir, los antecedentes que desarrolla un paciente son una forma importante de determinar el pronóstico de vida de ellos, y por ende la calidad de vida. (D. Rincóna, 2016).

\section{Metodología.}

El estudio se realizó en el Hospital Teodoro Maldonado Carbo, en el área de hospitalización del Servicio de Gastroenterología; la investigación se realizó a manera de un estudio de carácter observacional indirecto de enfoque cualitativo, de diseño no experimental, descriptivo, de corte retrospectivo y transversal. Los datos se obtuvieron de la revisión de las historias clínicas de pacientes hospitalizados, egresados o declarados como fallecidos, con diagnóstico de cirrosis hepática, dentro del período establecido desde enero 2014 hasta diciembre 2015; mediante colección de datos por fichas físicas y luego estas llevadas a digital en el Servicio de Estadística por el sistema AS-400.

Se obtuvo un universo de 1451 pacientes hospitalizados en el Servicio de Gastroenterología del Hospital Teodoro Maldonado Carbo; durante el período enero 2014 a diciembre 2015, con diagnóstico de cirrosis hepática; se tomó como muestra a 151 pacientes ingresados con diagnostico mencionado, sujeto a criterios de selección.

Se determinaron los criterios de inclusión para el estudio, seleccionando a pacientes ingresados en el área de hospitalización, con diagnostico confirmatorio de cirrosis hepática, independiente de la etiología, con datos de laboratorio e historia clínica completa, donde se mencione su valoración clínica, estadio de la cirrosis y pronóstico. Se excluyeron aquellos pacientes que no presentaban una causa definida descrita de cirrosis y a los pacientes que fallecieron sin definir causa a pesar del diagnóstico de cirrosis.

Se operacionalizaron las siguientes variables: edad, sexo, etnia, escolaridad, conductas adictivas de los pacientes (tabaco, alcohol, tipo de dieta: hiperproteica, hipoproteica, normocalórica), comorbilidades (hipertensión arterial, diabetes tipo 2, nefropatia, hipertensión portal). Además de valoración clínica de la cirrosis (estadio clínico I, II, III, IV), estado compensado o descompensado, y calidad de vida, mediante el empleo de la escala MELD y Child-Pugh. 


\section{Resultados.}

El 39\% de ellos fueron femeninas y el 61\% Masculinos, 59 y 92 pacientes respectivamente, se evidencia predominancia de la enfermedad en el sexo masculino.

Tabla 1. Sexo de la población estudiada.

\begin{tabular}{lll}
\hline Sexo & Número & Porcentaje \\
\hline HOMBRES & 92 & $60.93 \%$ \\
MUJERES & 59 & $39,07 \%$ \\
TOTAL & 151 & $100,00 \%$ \\
\hline
\end{tabular}

Fuente: Hospital Teodoro Maldonado Carbo.

Elaboración: Grupo de investigación.

La edad mínima fue de 33 años y la máxima con 88; con una mediana de 66 y moda de 67, hay una mayor tendencia de cirrosis en pacientes adultos mayores.

Tabla 2. Medidas de tendencia central en relación a la edad.

\begin{tabular}{ll}
\hline $\begin{array}{l}\text { MEDIDAS DE } \\
\text { TENDENCIA } \\
\text { CENTRAL }\end{array}$ & VALOR \\
\hline MINIMA & \\
MEDIA & 33 \\
MEDIANA & 64,8 \\
MODA & 66 \\
MAXIMA & 67 \\
\hline
\end{tabular}

Fuente: Hospital Teodoro Maldonado Carbo.

Elaboración: Grupo de investigación.

La gran mayoría de los pacientes comprendía entre 63 y 77 años, con una frecuencia de 71, de los cuales 40 eran hombres y 31 mujeres. Se mantiene la predominancia en el sexo masculino. 
Tabla 3. Rango de edad.

\begin{tabular}{lllll}
\hline RANGO MAXIMO & HOMBRES & MUJERES & NÚMERO & PORCENTAJE \\
\hline $\mathbf{3 3}-\mathbf{4 7}$ & 10 & 2 & 12 & $7.95 \%$ \\
$\mathbf{4 8}-\mathbf{6 2}$ & 25 & 16 & 41 & $27,15 \%$ \\
$\mathbf{6 3}-\mathbf{7 7}$ & 40 & 31 & 71 & $47.02 \%$ \\
$\mathbf{7 8}-\mathbf{9 2}$ & 17 & 10 & 27 & $17.88 \%$ \\
TOTAL & 92 & 59 & 151 & $100,00 \%$
\end{tabular}

Fuente: Hospital Teodoro Maldonado Carbo.

Elaboración: Grupo de investigación.

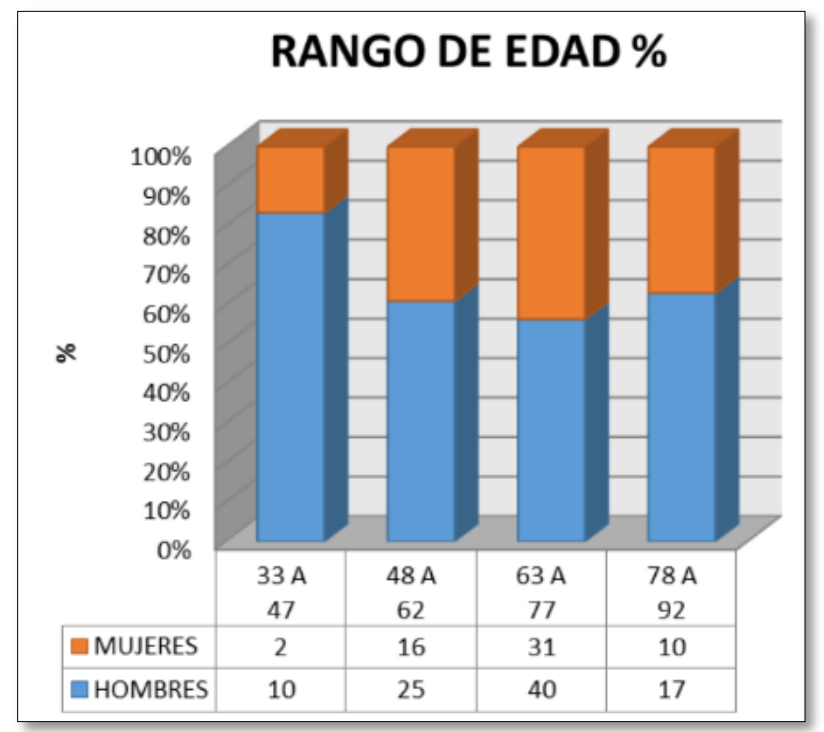

Gráfico 1. Rango de edad.

Fuente: Hospital Teodoro Maldonado Carbo.

Elaboración: Grupo de investigación.

46.36\% de los pacientes presentaron antecedentes cardiovasculares (entre ellos IAM e HT como más frecuentes), seguidos de enfermedades metabólicas (DM) y finalmente gastroenterológicas (la mayoría: sangrado digestivo alto y gastritis).

Tabla 4. Antecedentes patológicos de los pacientes seleccionados.

\begin{tabular}{lll}
\hline $\begin{array}{l}\text { ANTECEDENTES } \\
\text { PATOLÓGICOS }\end{array}$ & NÚMERO & PORCENTAJE \\
\hline NEUROLÓGICO & 1 & $0,66 \%$ \\
\hline
\end{tabular}




\begin{tabular}{lll}
\hline OCULAR & 1 & $0,66 \%$ \\
DERMATOLÓGICAS & 2 & $1,32 \%$ \\
PSICOLÓGICOS & 3 & $1,99 \%$ \\
HEMATOLÓGICO & 3 & $1,99 \%$ \\
HIPERTENSIÓN PORTAL & 6 & $3,97 \%$ \\
OSEO & 6 & $3,97 \%$ \\
PULMONAR & 6 & $3,97 \%$ \\
RENAL & 14 & $9,27 \%$ \\
GASTROENTEROLÓGICAS & 55 & $36,42 \%$ \\
METABÓLICAS & 62 & $41,06 \%$ \\
CARDIOVASCULARES & 70 & $46,36 \%$ \\
CIRROSIS & 151 & $100,00 \%$ \\
TOTAL PACIENTES & 151 & $100,00 \%$
\end{tabular}

Fuente: Hospital Teodoro Maldonado Carbo.

Elaboración: Grupo de investigación.

Un gran número de ellos con más del $50 \%$ ya habían sido sometidos a procedimientos gastroenterológicos: la más predominante fue ligaduras de varices esofágicas, correlacionado a los altos casos de APP: sangrado digestivo alto.

Tabla 5. Antecedentes quirúrgicos de los pacientes seleccionados.

\begin{tabular}{lll}
\hline $\begin{array}{l}\text { ANTECEDENTES } \\
\text { QUIRÚRGICOS }\end{array}$ & NÚMERO & PORCENTAJE \\
\hline CARDIOVASCULAR & 3 & $1,99 \%$ \\
RENAL & 4 & $2,65 \%$ \\
N/R & 46 & $30,46 \%$ \\
GASTROENTEROLÓGICAS & 77 & $50,99 \%$ \\
TOTAL & 151 & $100,00 \%$ \\
\hline
\end{tabular}

Fuente: Hospital Teodoro Maldonado Carbo.

Elaboración: Grupo de investigación. 
El mayor número de pacientes corresponde a aquellos cuyos hábitos se vinculan con el alcoholismo, a pesar de haber otras variables en el susodicho gráfico, todas expresan que la causa más evidente es el alcoholismo. Hubo datos sesgados, por datos inconclusos en las historias clínicas (HC).

Tabla 6. Hábitos tóxicos de los pacientes.

\begin{tabular}{lll}
\hline HÁBITOS & NÚMERO & PORCENTAJE \\
\hline TABAQUISMO & 2 & $1,32 \%$ \\
$\begin{array}{l}\text { TABAQUISMO+DESORDENES } \\
\text { ALIMENTICIOS }\end{array}$ & 2 & $1,32 \%$ \\
$\begin{array}{l}\text { ALCOHOLISMO+DESORDENES } \\
\text { ALIMENTICIOS }\end{array}$ & 6 & $3,97 \%$ \\
$\begin{array}{l}\text { ALCOHOLISMO + TABAQUISMO } \\
\text { DESORDENES ALIMENTICIOS }\end{array}$ & 10 & $6,62 \%$ \\
ALCOHOLISMO & 18 & $11,92 \%$ \\
N/R & 57 & $37,75 \%$ \\
TOTAL & 56 & $37,09 \%$ \\
\hline
\end{tabular}

Fuente: Hospital Teodoro Maldonado Carbo.

Elaboración: Grupo de investigación.

El mayor número de pacientes presentaba hábitos de 3 cruces, indicando que son consumidores habituales (más de tres veces en una semana), abarcando el $32 \%$, los datos 2 cruz (hasta tres veces por semana) en $12 \%$ y 1 cruz (menos de 1 vez por mes) en $5 \%$. Hubo datos sesgados de consumo en pacientes con antecedentes por $\mathrm{HC}$ con datos inconclusos.

Tabla 7. Hábitos del alcohol de los pacientes.

\begin{tabular}{lll}
\hline ALCOHOLISMO & NÚMERO & PORCENTAJE \\
\hline 2 CRUZ & 18 & $11,92 \%$ \\
3 CRUZ & 49 & $32,45 \%$ \\
$\mathbf{4}$ CRUZ & 7 & $4,64 \%$ \\
N/R & 77 & $50,99 \%$ \\
TOTAL & 151 & $100,00 \%$ \\
\hline
\end{tabular}

Fuente: Hospital Teodoro Maldonado Carbo.

Elaboración: Grupo de investigación. 
El síndrome ascítico es la complicación más frecuente en la muestra, con un $40 \%$, estos pacientes ya pronosticaban un pronóstico malo (todos fallecieron alrededor de un año posterior a la presentación del síndrome). La hipertensión portal se convirtió en la más frecuente complicación registrada, en pacientes con APQX con un $22 \%$.

Tabla 8. Complicaciones presentadas en los pacientes cirróticos.

\begin{tabular}{lll}
\hline COMPLICACIONES & NÚMERO & PORCENTAJE \\
\hline COLANGITIS & 21 & $13,91 \%$ \\
ENCEFALOPATÍA HEPÁTICA & 19 & $12,58 \%$ \\
HEMORRAGIA DIGESTIVA ALTA & 33 & $21,85 \%$ \\
INSUFICIENCIA HEPÁTICA & 12 & $7,95 \%$ \\
TERMINAL & & \\
SÍNDROME ASCÍTICO & 60 & $39,74 \%$ \\
SÍNDROME & & \\
HEPATORENAL/HEPATOPULMONAR & 6 & $3,97 \%$ \\
TOTAL & 151 & $100,00 \%$ \\
\hline
\end{tabular}

Fuente: Hospital Teodoro Maldonado Carbo.

Elaboración: Grupo de investigación.

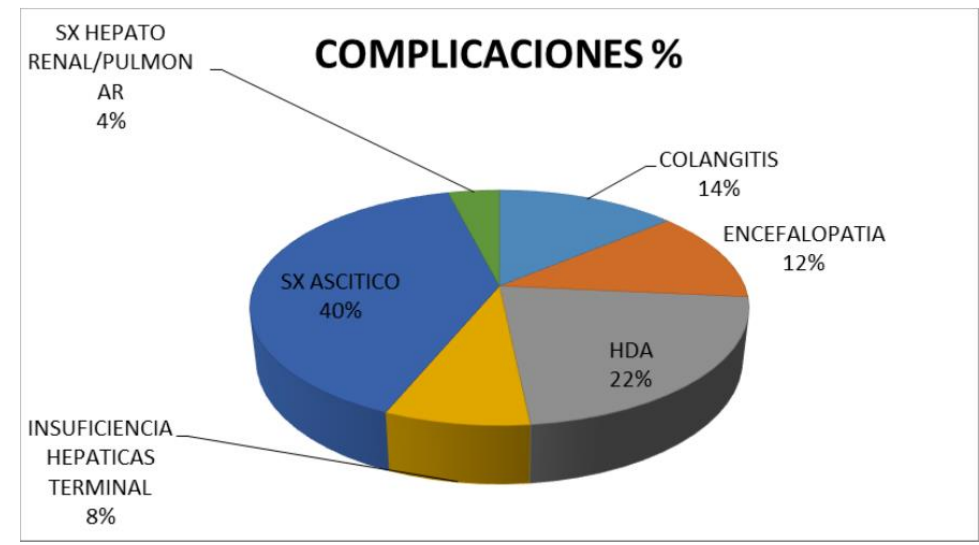

Gráfico 2. Complicaciones presentadas en los pacientes cirróticos. Fuente: Hospital Teodoro Maldonado Carbo. Elaboración: Grupo de investigación.

El $15.89 \%$ de la muestra se encuentra con clasificación A, refiere que su estado hemodinámico es más estable y su sobrevida en 2 años llega al 85\%; con clasificación B el 
$37.75 \%$ y su sobrevida en 2 años llega al 60\%; y el $46.36 \%$ con clasificación C y su sobrevida en 2 años llega al 30\%; indicando mayor grado de mortalidad conforme avanza la estatificación. Sumando los estadios B y C, se alcanza un total de $84,11 \%$ de la muestra.

Tabla 9. Distribución de los pacientes según Escala de Child-Pugh.

\begin{tabular}{lccl}
\hline ESCALA & NÚMERO & PORCENTAJE & $\begin{array}{l}\text { PROBABILIDAD } \\
\text { DE SOBREVIDA } \\
\text { EN 2 ANOS. }\end{array}$ \\
\hline CHILD PUGH A & & & $85 \%$ \\
CHILD PUGH B & 24 & $15,89 \%$ & $60 \%$ \\
CHILD PUGH C & 57 & $37,75 \%$ & $35 \%$ \\
TOTAL & 70 & $46,36 \%$ & \\
\hline
\end{tabular}

Fuente: Hospital Teodoro Maldonado Carbo.

Elaboración: Grupo de investigación.

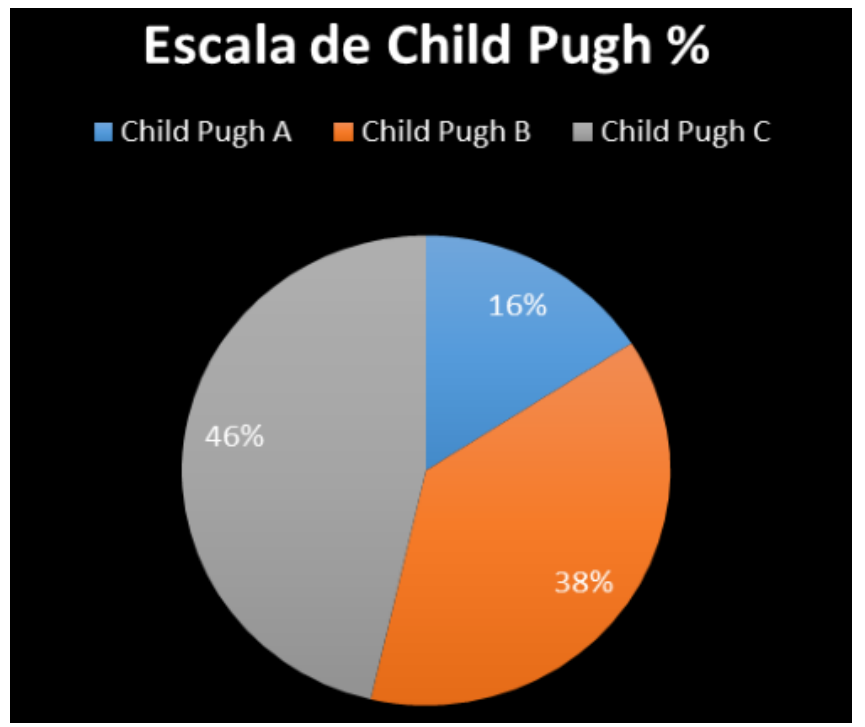

Gráfico 3. Distribución de los pacientes según Escala de Child-Pugh. Fuente: Hospital Teodoro Maldonado Carbo. Elaboración: Grupo de investigación.

70 pacientes presentaron clasificación entre 10-19 con la escala de MELD, representando el $46.36 \%$ de ellos. Un MELD con puntaje menor a 9 tienen una sobrevida a los 3 meses de 71,3; un MELD con 10 a 19 tienen una sobre vida a los 3 meses de 52,6\%; un MELD de 20 a 29 tienen una sobre vida a los 3 meses de 19,6; un MELD de 30 a 39 tienen una sobrevida 
a los 3 meses del 6\%; mientras que un MELD con más de 40 tiene de apenas 1,2\% de sobrevida en 3 meses.

Tabla 10. Probabilidad de mortalidad en 3 meses de los pacientes según Escala MELD.

\begin{tabular}{llll}
\hline ESCALA & NÚMERO & PORCENTAJE & $\begin{array}{l}\text { PROBABILIDAD } \\
\text { DE MORTALIDAD } \\
\text { EN 3 MESES. }\end{array}$ \\
\hline MELD MENOR A 9 & & & $71,3 \%$ \\
MELD 10 - 19 & 17 & $11,26 \%$ & $52,6 \%$ \\
MELD 20 - 29 & 70 & $46,36 \%$ & $19,6 \%$ \\
MELD 30 - 39 & 41 & $27,15 \%$ & $6 \%$ \\
MELD 40 O MÁS & 17 & $11,26 \%$ & $1,9 \%$ \\
TOTAL & 6 & $3,97 \%$ &
\end{tabular}

Fuente: Hospital Teodoro Maldonado Carbo.

Elaboración: Grupo de investigación.

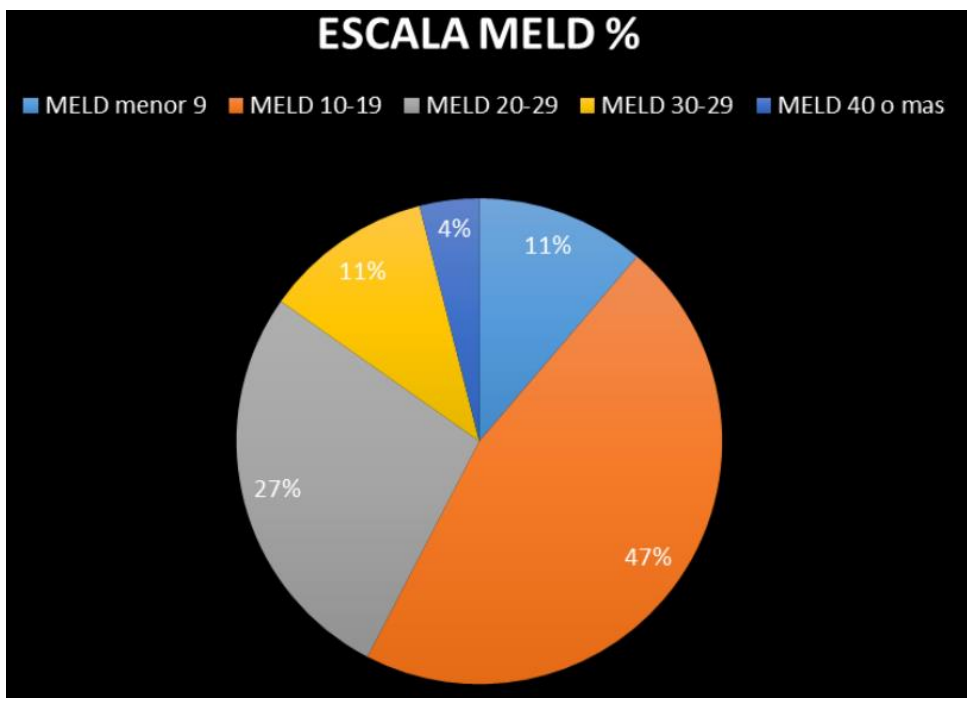

Gráfico 4. Distribución de los pacientes según Escala de Child-Pugh. Fuente: Hospital Teodoro Maldonado Carbo.

Elaboración: Grupo de investigación. 


\section{Conclusiones.}

- En relación a la edad tenemos que la edad mínima fue de 33 años y la máxima con 88. La gran mayoría tenía 67 años con una media de 66. Un gran porcentaje de ellos comprendía edades entre 63 y 77 años, dando una curva con área de dispersión centrada a la media.

- Como antecedentes patológicos personales primaron los cardiovasculares entre ellos Hipertensión, IAM y ACV (todos ellos con el $46.36 \%$ de frecuencia), seguidos por antecedentes metabólicos (DM). Mientras que quirúrgicamente el 51\% ya habían sido cometidos a procedimientos gastroenterológicos como ligaduras de varices esofágicas

sangrentes. En cuanto a los hábitos de los pacientes, el $37.75 \%$ de ellos tenía antecedentes de ingesta de alcohol +++ y el $11.92 \%$ con desórdenes alimenticios de tipo nutricional

- Como complicaciones debemos mencionar que el $100 \%$ de ellos ingreso con diagnóstico de descompensación por hepatopatía. Donde el 39.74\% había ya realizado ascitis y el 21.85\% Hemorragia Digestiva Alta.

- Basados en la escala de Child Pugh, 24 de ellos tenía un marcador A que representa el $15.89 \%$; tipo B con 57 pacientes representando el 37.75\%; tipo C con 70 pacientes representa el $46.36 \%$. Es decir, la tendencia de cirrosis hepática se clasifica en estadios avanzados con compromiso hepático mayor con una supervivencia en 2años de apenas el 35\%.

- Mediante la escala valorativa con MELD, menor a 9 tenemos 17 pacientes con 11.26\%; de 10-19 de puntuación 70 pacientes representan el 46.36\%; de 20-39 con 41 pacientes representan $27.15 \%$; de $30-39$ con 17 pacientes representando el $11.26 \%$ y con más de 40 en puntuación a 6 pacientes con 3.97\%. Vemos como la tendencia de supervivencia se localiza más entre los comprendidos entre 10-19 con puntuación MELD que corresponde a una mortalidad del $52.6 \%$

\section{Referencias bibliográficas.}

Bueya, L. G., \& Mateosb, F. G. (2012). Cirrosis hepática. Medicine., 625-33.

D. Rincóna, R. B. (2016). Cirrosis hepática. Medicine., 12(11):597-605.

García-Martínez, R. (2016). Ascitis, insuficiencia renal aguda y encefalopatía hepática. Medicine, 617-26.

L. Téllez*, M. A.-R.-G. (2016). Ascitis: diagnóstico diferencial y tratamiento. Medicine., $673-82$ 
Michael Malinchoc. (2000). A Model to Predict Poor Survival in Patients Undergoing Transjugular Intrahepatic Portosystemic Shunts. HEPATOLOGY , Vol. 31, No. $4 .$.

Moncayo, D. J. (2014). Compendio anual INEC. INEC, 65.

Octavio Campollo, M. M.-S.-A. (1997,). Características epidemiológicas de la cirrosis hepática en el Hospital Civil de Guadalajara. Salud Pública de México, vol.39, no.3.28-31.

Phillip S. Ge, M. a. (2016). Treatment of Patients with Cirrhosis. nejm.org, 375;8.

Phillip S. Ge, M. a. (August 25, 2016). Treatment of Patients with Cirrhosis. NEJM, 767778-2632.

Robert S. O'Shea, S. D. (2010). Alcoholic Liver Disease. AASLD PRACTICE GUIDELINES, 307.

Shah, S. A. (2012). Comparison of Maddrey Discriminant Function, Child-Pugh Score and Glasgow Alcoholic Hepatitis Score in predicting 28-day mortality on admission in patients with acute hepatitis. Royal Academy of Medicine in Ireland.

Usiña, J., \& Carrera, S. (2013). Anuario de estadísticas vitales nacimientos y defunciones 2013. DIRECCIÓN DE ESTADÍSTICAS SOCIDEMOGRÁFICAS, 1-527.

web, I. (2016). Inec.gob.ec. Recuperado el 25 de Octubre de 2016, de Inec.gob.ec: http://www.inec.gob.ec/inec/index.php?option=com_content $\&$ view=article $\& i d=61$ \%3Amas-de-900-mil-ecuatorianos-consumen alcohol $\&$ catid $=56 \% 3$ Adestacados $\&$ Itemid $=3 \&$.

\section{Ligital}




\section{PARA CITAR EL ARTÍCULO INDEXADO.}

Flores Poveda, K. A., Morante Arias, J. E., Flores Subia, D., \& Méndez Castro, A. (2019). Cirrosis hepática: perfil epidemiológico y calidad de vida. Hospital Teodoro Maldonado Carbo. Período 2014 - 2015. Ciencia Digital, 3(4), 82-100. https://doi.org/10.33262/cienciadigital.v3i4.936

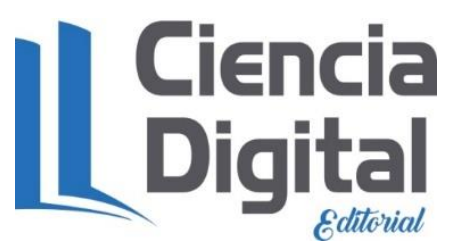

El artículo que se publica es de exclusiva responsabilidad de los autores y no necesariamente reflejan el pensamiento de la Revista Ciencia Digital.

El artículo queda en propiedad de la revista y, por tanto, su publicación parcial y/o total en otro medio tiene que ser autorizado por el director de la Revista Ciencia Digital.
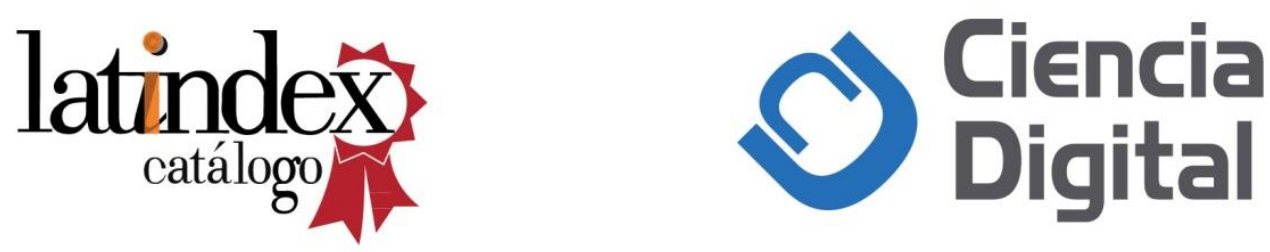